\title{
ON SOFT LIFTS AND SOFT TRANSFORMATIONS INDUCED BY SOFT SETS
}

\author{
Young Key Kim ${ }^{1}$, Won Keun $\mathrm{Min}^{2}$ \\ ${ }^{1}$ Department of Mathematics \\ MyongJi University \\ Youngin, 449-728, KOREA \\ ${ }^{2}$ Department of Mathematics \\ Kangwon National University \\ Chuncheon, 200-701, KOREA
}

\begin{abstract}
We introduced the notion of monotonic operation $u_{F}: P(X) \rightarrow P(X)$ induced by $A_{F}, F^{\leftarrow}$ in any soft set $(F, X)(A \subseteq X)$ in [13]. In this paper, we also introduce the notions of soft lift and soft transformation induced by $F^{\leftarrow}$. We investigate properties for the notions. In particular, Theorem 3.14 and Theorem 3.15 are obtained.
\end{abstract}

AMS Subject Classification: 94D05, 94D99, 03E70, 03E72

Key Words: soft sets, monotonic operation, quasi-open soft sets, topological universe

\section{Introduction}

We know that fuzzy sets [21], vague sets [9] and rough sets [18] are tools applied to avoid difficulties for dealing with uncertainties in economics, engineering, environmental science, sociology and computer science. In 1999, Molodtsov introduced the concept of soft set [17] to deal complicated problems and uncertainties as the following: The soft set is an approximate description of an object precisely consisting of two parts, namely predicate and approximate value set. In [14], Maji et al. introduced several operators for soft set theory: equality of

Received: November 13, 2015

Published: February 26, 2016

${ }^{\S}$ Correspondence author (c) 2016 Academic Publications, Ltd.

url: www.acadpubl.eu 
two soft sets, subset and superset of soft set, complement of a soft set, null soft set, and absolute soft set. For the soft set theory, Ali et al. [2] proposed new operations on soft sets, and in [4], the new operations were investigated with the notions defined in $[2,14]$. Recently, the properties and applications on the soft set theory have been studied widely and applied in the various branches of mathematics $[1,3,4,5,6,7,8,10,11,15,16,17,20]$. In particular, the combining the soft set theory and any other notions of mathematics have been studied steadily. In $[12,16]$, we combined topological structures and soft set theory. In this paper, we are going to combine a monotonic operation and soft set theory. We introduced the notion of monotonic operation $u_{F}: P(X) \rightarrow P(X)$ induced by $A_{F}, F^{\leftarrow}[12,16]$ in a given soft set $(F, X)(A \subseteq X)$ where $U$ is a common universe set and $X$ is a fixed subset of parameters, and studied basic properties of the operation. In this paper, we also introduce the notions of soft lift and soft transformation induced by $F^{\leftarrow}$. We investigate properties for the notions and the relations among soft lifts, soft transformations and the operation $u_{F}$. Finally, we have Theorem 3.14 and Theorem 3.15.

\section{Preliminaries}

Let $U$ be an initial universe set (simply, universe set) and $E$ be a collection of all possible parameters with respect to $U$, where parameters are the characteristics or properties of objects in $U$. We will call $E$ the set of parameters with respect to $U$.

Definition 2.1 ([17]). A pair $(F, A)$ is called a soft set over $U$ if $A \subset E$ and $F: A \rightarrow P(U)$, where $P(U)$ is the set of all subsets of $U$.

Definition $2.2([14])$. Let $U$ be a universe set and $E$ be a set of parameters. Let $(F, A)$ and $(G, B)$ be soft sets over a common universe set $U$ and $A, B \subseteq E$. Then $(F, A)$ is a subset of $(G, B)$, denoted by $(F, A) \widetilde{\subseteq}(G, B)$, if

(i) $A \subset B$; (ii) for all $e \in A, F(e) \subseteq G(e)$.

$(F, A)$ equals $(G, B)$, denoted by $(F, A)=(G, B)$, if $(F, A) \widetilde{\subseteq}(G, B)$ and $(G, B) \widetilde{\widetilde{\subseteq}}(F, A)$.

Definition $2.3([14])$. A soft set $(F, A)$ over $U$ is said to be a null soft set denoted by $\Phi$, if $\forall e \in A, F(e)=\emptyset$.

Definition $2.4([14])$. A soft set $(F, A)$ over $U$ is said to be an absolute soft set denoted by $\tilde{A}$, if $\forall e \in A, F(e)=U$. 
Definition $2.5([16])$. Let $(F, X)$ be a soft set over a universe set $U$. For $A \subseteq X$, we define $F(A)=\cup\{F(a): a \in A\}$.

Definition $2.6([16])$. Let $(F, X)$ be a soft set over a universe set $U$. For $A \subseteq X \subseteq E$ and $S \subseteq U$,

$$
\begin{gathered}
A_{F}=\{a \in A: F(a)=\emptyset\} ; \quad A^{F}=\{a \in A: F(a) \neq \emptyset\} ; \\
F^{\leftarrow}(S)=\{a \in X: F(a) \subseteq S \text { and } F(a) \neq \emptyset\} .
\end{gathered}
$$

Lemma $2.7([16])$. Let $(F, X)$ be a soft set over a universe set $U$. Then for $A, B \subseteq X$,

(i) $A \subseteq B$ implies $F(A) \subseteq F(B)$; (ii) $F(A \cup B)=F(A) \cup F(B)$;

(iii) $F(A \cap B) \subseteq F(A) \cap F(B)$.

Theorem $2.8([12,16])$. Let $(F, X)$ be a soft set over a universe set $U$. Then for $A, B \subseteq X$,

(i) $A=A_{F} \cup A^{F}$; (ii) $F(A)=F\left(A^{F}\right)$; (iii) $A^{F} \subseteq F^{\leftarrow}(F(A))$;

(iv) if $A \subseteq B$, then $A_{F} \subseteq B_{F}, A^{F} \subseteq B^{F}$ and $F(A) \subseteq F(B)$;

(v) $F^{\leftarrow}\left(F\left(F^{\leftarrow}(F(A))\right)=F^{\leftarrow}(F(A))\right.$.

Let $(F, X)$ be a soft set over a universe set $U$. Let us define an operation $u_{F}: P(X) \rightarrow P(X)[13]$ as the following:

$$
u_{F}(A)=A_{F} \cup F^{\leftarrow}(F(A)) \quad \text { for } \quad A \in P(X) .
$$

Theorem 2.9 ([13]). Let $(F, X)$ be a soft set over a universe set $U$. Then we have the following things: For $A, B \subseteq X$,

(i) $u_{F}(\emptyset)=\emptyset$; (ii) $A \subseteq u_{F}(A)$; (iii) if $A \subseteq B$, then $u_{F}(A) \subseteq u_{F}(B)$;

(iv) $u_{F}(A)=u_{F}\left(u_{F}(A)\right)$.

\section{Main Results}

In this section, we introduce the notions of soft lifts and soft transformation, and investigate properties for such notions. Let $U$ be a universe set and $E$ be a set of parameters. Let us denote

$$
\mathcal{S} S_{X}=\{(F, X) \mid(F, X) \text { is a soft set for the fixed subset }
$$

$X$ over a universe set $U\}$. 
Lemma 3.1. Let $(F, X) \in \mathcal{S} S_{X}$ and $V_{1}, V_{2} \subseteq U$. Then we have the following things:

(i) $F^{\leftarrow}\left(V_{1}\right) \cap F^{\leftarrow}\left(V_{2}\right)=F^{\leftarrow}\left(V_{1} \cap V_{2}\right)$.

(ii) $F^{\leftarrow}\left(V_{1}\right) \cup F^{\leftarrow}\left(V_{2}\right) \subseteq F^{\leftarrow}\left(V_{1} \cup V_{2}\right)$.

Proof. (i) For $a \in X, a \in F^{\leftarrow}\left(V_{1}\right) \cap F^{\leftarrow}\left(V_{2}\right)$ iff $a \in F^{\leftarrow}\left(V_{1}\right)$ and $a \in F^{\leftarrow}\left(V_{2}\right)$ iff $F(a) \subseteq V_{1}$ and $F(a) \subseteq V_{2}$ for $F(a) \neq \emptyset$ iff $F(a) \subseteq V_{1} \cap V_{2}$ for $F(a) \neq \emptyset$ iff $a \in F^{\leftarrow}\left(V_{1} \cap V_{2}\right)$.

(ii) For $a \in X, a \in F^{\leftarrow}\left(V_{1}\right) \cup F^{\leftarrow}\left(V_{2}\right) \Rightarrow a \in F^{\leftarrow}\left(V_{1}\right)$ or $a \in F^{\leftarrow}\left(V_{2}\right) \Rightarrow$ $F(a) \subseteq V_{1}$ or $F(a) \subseteq V_{2}$ for $F(a) \neq \emptyset \Rightarrow F(a) \subseteq V_{1} \cup V_{2}$ for $F(a) \neq \emptyset \Rightarrow$ $a \in F^{\leftarrow}\left(V_{1} \cup V_{2}\right)$.

Example 3.2. Let $U=\left\{x_{1}, x_{2}, x_{3}, x_{4}\right\}$ and $E=\left\{e_{1}, e_{2}, e_{3}, e_{4}\right\}$. Consider $X=\left\{e_{1}, e_{2}, e_{3}\right\}$ and a soft set $(F, X)$ defined as the following:

$$
F\left(e_{1}\right)=\emptyset ; F\left(e_{2}\right)=\left\{x_{2}\right\} ; \quad F\left(e_{3}\right)=\left\{x_{1}, x_{3}\right\} .
$$

Let $V_{1}=\left\{x_{1}, x_{2}\right\}$ and $V_{2}=\left\{x_{2}, x_{3}, x_{4}\right\}$. Then $F^{\leftarrow}\left(V_{1} \cup V_{2}\right)=\left\{e_{2}, e_{3}\right\}$. Note that $F\left(e_{3}\right) \nsubseteq V_{1}, V_{2}$. So $e_{3} \notin F^{\leftarrow}\left(V_{1}\right)$ and $e_{3} \notin F^{\leftarrow}\left(V_{2}\right)$. It implies that $F^{\leftarrow}\left(V_{1} \cup V_{2}\right) \neq F^{\leftarrow}\left(V_{1}\right) \cup F^{\leftarrow}\left(V_{2}\right)$.

Lemma 3.3. Let $(F, X) \in \mathcal{S} S_{X}$ and $x \in X$. Then $x \in F^{\leftarrow}(F(x))$ if and only if $F(x) \neq \emptyset$.

Proof. Obvious.

Definition 3.4. Let $(F, X) \in \mathcal{S} S_{X}$. Let us define a mapping $\varphi_{F}: X \rightarrow$ $P(X)$ by $\varphi_{F}(x)=F^{\leftarrow}(F(x))$ for each $x \in X$. Then we call the mapping $\varphi_{F}$ a soft lift over $X$ induced by $(F, X)$. We define $\varphi_{F}(A)=\cup\left\{\varphi_{F}(a): a \in A\right\}$.

Theorem 3.5. Let $(F, X) \in \mathcal{S} S_{X}$ and $x \in X$. Then $\varphi_{F}\left(\varphi_{F}(x)\right)=\varphi_{F}(x)$.

Proof. First, we show that $\varphi_{F}\left(\varphi_{F}(x)\right) \subseteq \varphi_{F}(x)$. From Lemma 3.1 and Theorem 2.8,

$$
\begin{aligned}
\varphi_{F}\left(\varphi_{F}(x)\right) & =\varphi_{F}\left(F^{\leftarrow}(F(x))\right)=\cup_{z \in F^{\leftarrow}(F(x))} \varphi_{F}(z) \\
& =\cup_{z \in F^{\leftarrow}(F(x))} F^{\leftarrow}(F(z)) \subseteq F^{\leftarrow}\left(\cup_{z \in F^{\leftarrow}(F(x))} F(z)\right) \\
& =F^{\leftarrow}\left(F\left(F^{\leftarrow}(F(x))\right)\right)=F^{\leftarrow}(F(x)) \\
& =\varphi_{F}(x) .
\end{aligned}
$$

For the other inclusion, let $z \in \varphi_{F}(x)=F^{\leftarrow}(F(x))$; then $F(z) \neq \emptyset$ and so by Lemma 3.3, $z \in F^{\leftarrow}(F(z))$ and $z \in \varphi_{F}(z)$ for $z \in F^{\leftarrow}(F(x))$. It implies that $z \in \varphi_{F}\left(F^{\leftarrow}(F(x))\right)=\varphi_{F}\left(\varphi_{F}(x)\right)$. Hence, $\varphi_{F}(x) \subseteq \varphi_{F}\left(\varphi_{F}(x)\right)$. 
Theorem 3.6. Let $(F, X) \in \mathcal{S} S_{X}$ and $\varphi_{F}: X \rightarrow P(X)$ a soft lifting over $X$. Then for $A, B \subseteq X$,

(i) $\varphi_{F}(A \cup B)=\varphi_{F}(A) \cup \varphi_{F}(B)$;

(ii) if $A \subseteq B$, then $\varphi_{F}(A) \subseteq \varphi_{F}(B)$;

(iii) $\varphi_{F}(A \cap B) \subseteq \varphi_{F}(A) \cap \varphi_{F}(B)$;

(vi) $\varphi_{F}\left(\varphi_{F}(A)\right)=\varphi_{F}(A)$.

Proof. (i) Obvious.

(ii) It follows from (i).

(iii) It follows from (ii).

(vi) For $A \subseteq X$, from Theorem 3.5, it follows

$$
\varphi_{F}\left(\varphi_{F}(A)\right)=\varphi_{F}\left(\cup_{a \in A} \varphi_{F}(a)\right)=\cup_{a \in A} \varphi_{F}\left(\varphi_{F}(a)\right)=\cup_{a \in A} \varphi_{F}(a)=\varphi_{F}(A)
$$

So $\varphi_{F}\left(\varphi_{F}(A)\right)=\varphi_{F}(A)$.

Example 3.7. Let $U=\left\{x_{1}, x_{2}, x_{3}, x_{4}, x_{5}\right\}$ and $E=\left\{e_{1}, e_{2}, e_{3}, e_{4}\right\}$. Consider $X=E$ and a soft set $(F, X)$ defined as the following:

$$
F\left(e_{1}\right)=\left\{x_{1}, x_{2}\right\} ; \quad F\left(e_{2}\right)=\left\{x_{2}\right\} ; \quad F\left(e_{3}\right)=\left\{x_{2}, x_{3}\right\} ; \quad F\left(e_{4}\right)=\left\{x_{3}, x_{5}\right\} .
$$

Let $A=\left\{e_{1}\right\}$ and $B=\left\{e_{3}, e_{4}\right\}$. Note that:

$$
\begin{gathered}
\varphi_{F}(A)=F^{\leftarrow}\left(F\left(e_{1}\right)\right)=\left\{e_{1}, e_{2}\right\} ; \\
\varphi_{F}(B)=F^{\leftarrow}\left(F\left(e_{3}\right)\right) \cup F^{\leftarrow}\left(F\left(e_{4}\right)\right)=\left\{e_{2}, e_{3}, e_{4}\right\} ;
\end{gathered}
$$

Note that $\varphi_{F}(A) \cap \varphi_{F}(B)=\left\{e_{2}\right\}$ and $\varphi_{F}(A \cap B)=\emptyset$. So $\varphi_{F}(A \cap B) \neq$ $\varphi_{F}(A) \cap \varphi_{F}(B)$.

Theorem 3.8. Let $(F, X) \in \mathcal{S} S_{X}$ and $\varphi_{F}: X \rightarrow P(X)$ a soft lift over $X$. Then for $A \subseteq X$,

(i) $\varphi_{F}\left(A_{F}\right)=\emptyset$;

(ii) $A^{F} \subseteq \varphi_{F}\left(A^{F}\right)$;

(iii) $\varphi_{F}(A) \subseteq F^{\leftarrow} F(A)$;

(iv) $\varphi_{F}(A)=\varphi_{F}\left(A^{F}\right)$;

(v) $\varphi_{F}\left(F^{\leftarrow} F(A)\right)=F^{\leftarrow} F(A)$. 
Proof. (i) For each $a \in A_{F}$, since $F(a)=\emptyset$, obviously $\varphi_{F}\left(A_{F}\right)=\cup_{a \in A_{F}} \varphi_{F}(a)=$ $\emptyset$.

(ii) For each $x \in A^{F}$, by Lemma 3.3, $x \in F^{\leftarrow}(F(x))=\varphi_{F}(x) \subseteq \varphi_{F}\left(A_{F}\right)$. So we have (ii).

(iii) From (ii) of Lemma 3.1, $\varphi_{F}(A)=\cup\left\{\varphi_{F}(a): a \in A\right\}=\cup_{a \in A} F^{\leftarrow}(F(a)) \subseteq$ $F^{\leftarrow}\left(\cup_{a \in A} F(a)\right)=F^{\leftarrow} F(A)$.

(iv) From Theorem 2.8 and (i) of Theorem 3.6, $\varphi_{F}(A)=\varphi_{F}\left(A^{F} \cup A_{F}\right)=$ $\varphi_{F}\left(A^{F}\right) \cup \varphi_{F}\left(A_{F}\right)=\varphi_{F}\left(A^{F}\right)$.

(v) From $F^{\leftarrow}(F(A))=\left(F^{\leftarrow}(F(A))\right)^{F}$, (ii) and (iii), it follows $F^{\leftarrow}(F(A))=$ $\left(F^{\leftarrow}(F(A))\right)^{F} \subseteq \varphi_{F}\left(\left(F^{\leftarrow}(F(A))\right)^{F}\right)=\varphi_{F}\left(F^{\leftarrow}(F(A))\right) \subseteq F^{\leftarrow} F\left(F^{\leftarrow}(F(A))\right)=$ $F^{\leftarrow}(F(A))$. Finally, we have $\varphi_{F}\left(F^{\leftarrow}(F(A))\right)=F^{\leftarrow}(F(A))$.

Theorem 3.9. Let $(F, X) \in \mathcal{S} S_{X}$ and $(G, Y) \in \mathcal{S} S_{Y}$, and let $\varphi_{F}$ and $\psi_{G}$ be soft lifts over $X$ and $Y$, respectively. Then

(i) $\mu_{F} \circ \varphi_{F}=\varphi_{F}$;

(ii) $\mu_{G} \circ \psi_{G}=\psi_{G}$.

Proof. (i) For $x \in X$, from Theorem 2.8 and $\left(F^{\leftarrow}(F(x))\right)_{F}=\emptyset$, it follows

$$
\begin{aligned}
\left(\mu_{F} \circ \varphi_{F}\right)(x) & =\mu_{F}\left(\varphi_{F}(x)\right) \\
& =\mu_{F}\left(F^{\leftarrow}(F(x))\right) \\
& =F^{\leftarrow}\left(F\left(F^{\leftarrow}(F(x))\right) \cup\left(F^{\leftarrow}(F(x))\right)_{F}\right. \\
& =F^{\leftarrow}(F(x)) \\
& =\varphi_{F}(x)
\end{aligned}
$$

(ii) It is similar to the proof of (i).

Definition 3.10. Let $X$ and $Y$ be nonempty sets. We call the mapping $T: P(X) \rightarrow P(Y)$ a transformation if it satisfies the following axioms:

(i) $T(A)=\emptyset$ if and only if $A=\emptyset$.

(ii) For $A_{1}, A_{2} \in P(X), T\left(A_{1} \cup A_{2}\right)=T\left(A_{1}\right) \cup T\left(A_{2}\right)$.

Definition 3.11. Let $(F, X) \in \mathcal{S} S_{X}$ and $(G, Y) \in \mathcal{S} S_{Y}$, and let $\varphi_{F}$ and $\psi_{G}$ be soft lifts over $X$ and $Y$, respectively. Let $f: X \rightarrow Y$ be a mapping. Then the pair $(f, T)$ is called a soft transformation if $T \circ \varphi_{F}=\psi_{G} \circ f$.

Theorem 3.12. Let $(F, X) \in \mathcal{S} S_{X}$ and $(G, Y) \in \mathcal{S} S_{Y}$, and let $\varphi_{F}$ and $\psi_{G}$ be soft lifts over $X$ and $Y$, respectively. Let $f: X \rightarrow Y$ be a mapping and $T$ a transformation. Then 
(i) $T \circ \mu_{F} \circ \varphi_{F}=T \circ \varphi_{F}$;

(ii) $\mu_{G} \circ \psi_{G} \circ f=\psi_{G} \circ f$;

(iii) if $(f, T)$ is a soft transformation, then $T \circ \mu_{F} \circ \varphi_{F}=\mu_{G} \circ \psi_{G} \circ f$.

Proof. (i) and (ii) are obtained by Theorem 3.9.

(iii) It is obvious from (i) and (ii).

Theorem 3.13. Let $(F, X) \in \mathcal{S} S_{X}$ and $(G, Y) \in \mathcal{S} S_{Y}$, and let $\varphi_{F}$ and $\psi_{G}$ be soft lifts over $X$ and $Y$, respectively. Let $f: X \rightarrow Y$ be a function. If $(f, T)$ is a soft transformation, then $\mu_{G} \circ T \circ \varphi_{F}=T \circ \varphi_{F}$.

Proof. For $x \in X,\left(T \circ \varphi_{F}\right)(x)=\left(T \circ \mu_{F} \circ \varphi_{F}\right)(x)=\left(\mu_{G} \circ \psi_{G} \circ f\right)(x)=$ $\left(\mu_{G} \circ T \circ \varphi_{F}\right)(x)$.

Theorem 3.14. Let $(F, X) \in \mathcal{S} S_{X}$ and $(G, Y) \in \mathcal{S} S_{Y}$, and let $\varphi_{F}$ and $\psi_{G}$ be soft lifts over $X$ and $Y$, respectively. Let $(f, T)$ be a soft transformation. Then for $A \subseteq X$,

(i) $\left(T \circ \varphi_{F}\right)(A)=\left(\psi_{G} \circ f\right)(A)$;

(ii) $\left(T \circ \varphi_{F}\right)\left(A_{F}\right)=\left(\psi_{G} \circ f\right)\left(A_{F}\right)=\emptyset$;

(iii) $\left(T \circ \varphi_{F}\right)(A)=\left(\psi_{G} \circ f\right)\left(A^{F}\right)$.

Proof. (i) From the property of transformation, we have

$$
\begin{aligned}
\left(T \circ \varphi_{F}\right)(A)=T\left(\varphi_{F}(A)\right)=T\left(\cup_{a \in A} \varphi_{F}(a)\right) & =\cup_{a \in A} T\left(\varphi_{F}(a)\right)=\cup_{a \in A} \psi_{G}(f(a)) \\
& =\psi_{G}\left(\cup_{a \in A} f(a)\right)=\left(\psi_{G} \circ f\right)(A) .
\end{aligned}
$$

(ii) From (i) of Theorem 3.8, $\left(\psi_{G} \circ f\right)\left(A_{F}\right)=\left(T \circ \varphi_{F}\right)\left(A_{F}\right)=T\left(\varphi_{F}\left(A_{F}\right)\right)=$ $T(\emptyset)=\emptyset$.

(iii) Since $A=A^{F} \cup A_{F}$ and $A^{F} \cap A_{F}=\emptyset$, by (i) and (ii),

$$
\begin{aligned}
\left(T \circ \varphi_{F}\right)(A)= & \left(T \circ \varphi_{F}\right)\left(A^{F} \cup A_{F}\right) \\
& =T\left(\varphi_{F}\left(A^{F}\right) \cup \varphi_{F}\left(A_{F}\right)\right)=T\left(\varphi_{F}\left(A^{F}\right)\right)=\left(\psi_{G} \circ f\right)\left(A^{F}\right) .
\end{aligned}
$$

Theorem 3.15. Let $(F, X) \in \mathcal{S} S_{X}$ and $(G, Y) \in \mathcal{S} S_{Y}$, and let $\varphi_{F}$ and $\psi_{G}$ be soft lifts over $X$ and $Y$, respectively. If $(f, T)$ is a soft transformation, then

(i) $f\left(A^{F}\right)=(f(A))^{G}$;

(ii) $f\left(A_{F}\right)=(f(A))_{G}$. 
Proof. (i) Let $y \in f\left(A^{F}\right)$. Then there exists $a \in A^{F}$ such that $f(a)=y$ and $F(a) \neq \emptyset$. Since $(f, T)$ is a soft transformation and $F(a) \neq \emptyset, \psi_{G}(y)=$ $\psi_{G}(f(a))=T(\varphi(a))=T\left(F^{\leftarrow}(F(a))\right) \neq \emptyset$, that is, $\psi_{G}(y)=G^{\leftarrow}(G(y)) \neq \emptyset$. It implies that $G(y) \neq \emptyset$ and $y \in\left(f\left(A^{F}\right)\right)^{G} \subseteq(f(A))^{G}$, and so $f\left(A^{F}\right) \subseteq(f(A))^{G}$.

For the other inclusion, let $y \in f(A)^{G}$. Then $G(y) \neq \emptyset$ and $y \in f(A)$. There exists $a \in A$ such that $f(a)=y$ and $G(f(a))=G(y) \neq \emptyset$. So by Lemma 3.3, $y=f(a) \in G^{\leftarrow}(G(f(a)))=\left(\psi_{G} \circ f\right)(a)=T\left(\varphi_{F}(a)\right)=T\left(F^{\leftarrow}(F(a))\right)$. Since $T\left(F^{\leftarrow}(F(a))\right) \neq \emptyset, F^{\leftarrow}(F(a)) \neq \emptyset$ and $F(a) \neq \emptyset$. It implies $a \in A^{F}$ and $y=f(a) \in f\left(A^{F}\right)$. Hence $(f(A))^{G} \subseteq f\left(A^{F}\right)$.

(ii) Let $y \in f\left(A_{F}\right)$. Then there exists $a \in A_{F}$ such that $f(a)=y$. It implies that $\psi_{G}(f(a))=\psi_{G}(y)$ and $F(a)=\emptyset$. Since $(f, T)$ is a soft transformation and $F^{\leftarrow}(F(a))=\emptyset, \psi_{G}(f(a))=T\left(\varphi_{F}(a)\right)=T\left(F^{\leftarrow}(F(a))\right)=\emptyset$. So $\psi_{G}(y)=$ $G^{\leftarrow}(G(y))=\emptyset$ and $G(y)=\emptyset$. It implies $y \in\left(f\left(A_{F}\right)\right)_{G} \subseteq(f(A))_{G}$. So $f\left(A_{F}\right) \subseteq$ $(f(A))_{G}$.

For the other part of the proof, let $y \in f(A)_{G}$. Then $G(y)=\emptyset$ and $y \in f(A)$. There exists $a \in A$ such that $G(f(a))=G(y)$ and $G(y)=\emptyset$. Since $(f, T)$ is a soft transformation and $G(y)=\emptyset, T\left(F^{\leftarrow}(F(a))\right)=T\left(\varphi_{F}(a)\right)=\left(\psi_{G} \circ f\right)(a)=$ $G^{\leftarrow}(G(f(a)))=\emptyset$. Since $T\left(F^{\leftarrow}(F(a))\right)=\emptyset, F^{\leftarrow}(F(a))=\emptyset$ and so $F(a)=\emptyset$. It implies $a \in A_{F}$ and $y=f(a) \in f\left(A_{F}\right)$. Hence $(f(A))_{G} \subseteq f\left(A_{F}\right)$.

\section{References}

[1] H. Aktas, N. Cagman, Soft sets and soft groups, Information Sciences, 177 (2007), 27262735 .

[2] M.I. Ali, F. Feng, X.Y. Liu, W.K. Min, M. Shabir, On some new operations in soft set theory, Computers and Mathematics with Applications, 57 (2009), 1547-1553.

[3] M.I. Ali, M. Shabir, M. Naz, Algebraic structures of soft sets associated with new operations, Computers and Mathematics with Applications, 61 (2011), 2647-2654.

[4] M.I. Ali, Another view on reduction of parameters in soft sets, Applied Soft Computing, 12 (2012), 1814-1821.

[5] F. Feng, X. Liu, V. Leoreanu-Fotea, Y.B. Jun, Soft sets and soft rough sets, Information Sciences, 181 (2011), 1125-1137.

[6] F. Feng, C.X. Li, B. Davvaz, M. Irfan Ali, Soft sets combined with fuzzy sets and rough sets: a tentative approach, Soft Comput., 14 (2010), 899-911.

[7] F. Feng, Y. B. Jun and X. Zhao, Soft semirings, Comput. Math. Appl., 56(10)(2008), 2621-2628.

[8] F. Feng, Y.M. Li, V. Leoreanu-Fotea, Application of level soft sets in decision making based on interval-valued fuzzy soft sets, Comput. Math. Appl., 60 (2010), 1756-1767. 
[9] W. L. Gau and D. J. Buehrer, Vague sets, IEEE trans. System Man Cybernet, 23(2) (1993), 610-614.

[10] S. Hussain and B. Ahmad, Some properties of soft topological spaces, Comput. Math. Appl., 62(2011), 4058-4067.

[11] Y.B. Jun, Soft BCK/BCI-algebras, Comput. Math. Appl., 56 (2008), 1408-1413.

[12] Y.K. Kim and W.K. Min, Remarks on Soft sets over a common topological universe, Far East Journal of Mathematical Sciences, 80(2)(2013), 169 - 179.

[13] Y.K. Kim and W.K. Min, Notes on a monotonic operation induced by soft sets, submitted.

[14] P.K. Maji, R. Biswas, R. Roy, Soft set theory, Comput. Math. Appl., 45 (2003), 555-562.

[15] W.K. Min, A note on soft topological spaces, Comput. Math. Appl., 62 (2011), 3524-3528.

[16] W.K. Min, Soft sets over a common topological universe, Journal of Intelligent and Fuzzy Systems, 26(5) (2014), 2099-2106.

[17] D. Molodtsov, Soft set theory first results, Computers and Mathematics with Applications, 37 (1999), 19-31.

[18] Z. Pawlak, Rough sets, International Journal of Computer and Information Sciences, 11 (1982), 341-356.

[19] M. Shabir and M. Naz, On soft topological spaces, Comput. Math. Appl., 61 (2011), 1786-1799.

[20] I. Zorlutuna, M. Akdag, W. K. Min, and S. Atmaca, Remarks on soft topological spaces, Annals of Fuzzy Mathematics and Informatics, 3 (2012), 171-185.

[21] L. A. Zadeh, Fuzzy sets, Inform. and Control, 8(1965), 338-353. 
